\title{
POSITIVE OPERATORS ON $C(X)$
}

\author{
S. R. FOGUEL ${ }^{1}$
}

ABstract. Conditions for the existence of finite and $\sigma$ finite invariant measures for a Markov operator on $C(X)$ are studied.

Let $X$ be a locally compact Hausdorff space and $\Sigma$ be the collection of Baire sets. Let $P$ be an operator on $C(X)$ which satisfies

(1) $\|P\|=1$,

(2) $P 1=1$.

Now if $f \geqq 0$ then $P f \geqq 0$, because we may assume that $0 \leqq f \leqq 1$ and then $P(1-f)=1-P f \leqq 1$.

We shall also assume:

(3) If $\mu$ is a countably additive measure then so is $P^{*} \mu$.

By a measure we mean a positive finite measure, unless otherwise stated. Every countably additive measure is regular since it is defined on Baire sets.

It is enough to assume (3) only for $\mu=\delta_{x}, x \in X$, since then $P(x, A)$ $=P^{*} \delta_{x}(A)$ is a probability measure for a fixed $x$ and if $A \in \Sigma, P(\cdot, A)$ is $\Sigma$ measurable: If $A \in \Sigma$ is compact then there exists a sequence of continuous functions $0 \leqq f_{n} \leqq 1, f_{n} \downarrow 1_{A}$ (where $1_{A}(x)=0$ if $x \notin A$ and 1 if $x \in A)$. Thus $P(x, A)=\lim _{n} P^{*} \delta_{x}\left(f_{n}\right)=\lim _{n} P f_{n}(x)$ and is measurable. Now the collection of $A \in \Sigma$ such that $P(\cdot, A)$ is measurable is a $\sigma$ field and thus all of $\Sigma$. Clearly $P^{*} \mu(A)=\int P(x, A) \mu(d x)$ is a countably additive measure whenever $\mu$ is. Also $P f(x)=\int P(x, d y) f(y)$ and if $0 \leqq f_{n} \leqq 1$ and $f_{n} \downarrow f$ then $P f_{n}(x) \rightarrow P f(x)$. This last property almost implies (3).

TheOREM 1. Let $X$ be a locally compact Hausdorff space and $X=\cup C_{n}$ where $C_{n}$ are compact Baire sets. If the operator $P$ satisfies (1), (2) and $\left(3^{\prime}\right)$ :

$\left(3^{\prime}\right)$ If $0 \leqq f_{n} \leqq 1, f_{n} \downarrow f$ and $f_{n}, f \in C(X)$ then $P f_{n}(x) \rightarrow P f(x)$.

Then condition (3) holds.

Proof. The operator $P^{*}$ is defined on finitely additive regular measures, by [1, Theorem IV.6.2]. Let $0 \leqq \mu$ be countably additive and put $P^{*} \mu=\nu_{0}+\nu_{1}$ as in [4, p. 52]; namely, $\nu_{1}$ is countably additive and $\nu_{0}$ is purely additive, i.e. if $0 \leqq \lambda \leqq \nu_{0}$ and $\lambda$ is countably additive then $\lambda=0$. Now if $A \in \Sigma$ is compact the restriction of $\nu_{0}$ to $A$ is countably additive by $\left[1\right.$, Theorem III. 5.13] and thus $\nu_{0}(A)=0$. Hence

Received by the editors August 30, 1968.

1 Supported by the National Science Foundation Grant GP-7475. 
$\nu_{0}(f)=0$ for every continuous function with compact support. There exists a sequence of functions $f_{n}, 0 \leqq f_{n} \leqq 1, f_{n}$ continuous with compact support, $f_{n} \geqq 1_{C_{n}}$. We may assume that the sequence is monotone since we may replace it by the sequence $\max \left(f_{1}, \cdots, f_{n}\right)$. Thus $f_{n} \uparrow 1$ and $\nu_{1}(X)=\lim \nu_{1}\left(f_{n}\right)=\lim P^{*} \mu\left(f_{n}\right)=\lim \mu\left(P f_{n}\right)$ and by $\left(3^{\prime}\right)$ the limit is $\mu(X)$. Thus $\nu_{0}(X)=0$ and $\nu_{0}=0$.

REMARKs. If $X$ is compact then ( $\left.3^{\prime}\right)$ follows from Dini's Theorem. We do not know if $\left(3^{\prime}\right)$ suffices without assuming that $X$ is the union of countably many compact sets.

In $[2$, Theorem 1] the problem of existence of a finite measure was studied. This result can be improved.

Theorem 2. Assume (1), (2) and (3) and let $A$ be a compact Baire set. Then either

(a) $\sup _{x}(1 / N) \sum_{n=0}^{N-1} P^{n} 1_{A}(x) \rightarrow 0$ or

(b) there exists a countably additive invariant measure that does not vanish on $A$.

Proof. Assume that (a) is false. Let $\delta>0$ and $N_{i}$ be an infinite sequence such that

$$
A_{N_{i}}=\left\{x: \frac{1}{N_{i}} \sum_{n=0}^{N_{i}-1} P^{n} 1_{A}(x)>\delta\right\}
$$

are not empty. Put $\mu_{i}=\delta_{x_{i}}, x_{i} \in A_{N_{i}}$ and

$$
\lambda_{i}=\frac{1}{N_{i}} \sum_{n=0}^{N_{i}-1} P^{* n} \mu_{i}
$$

Then $\lambda_{i}$ is countably additive $0 \leqq \lambda_{i}, \lambda_{i}(X)=1$ and $\lambda_{i}(A) \geqq \delta$. Now any weak $*$ limit of $\lambda_{i}$ is an invariant measure that does not vanish on $A$.

To conclude this paper let us consider the sufficient condition, for the existence of a $\sigma$ finite invariant measure studied in [3]. Put $T_{A} f(x)=1_{A}(x) f(x)$ then, by [3], an invariant $\sigma$ finite measure exists if for some Baire set $A$, whose complement is compact,

(4) $\left(T_{A} P T_{A}\right)^{n} 1(x) \rightarrow 0$ for every $x \in X$, as $n \rightarrow \infty$. Also the invariant measure is finite on every compact set.

Let $u$ be the collection of all open Baire sets whose closure is compact.

Lemma 1. Condition (4) holds for every set $A$ whose complement $B$ is the closure of a set in $\mathcal{u}$ if and only if condition (4) holds for every set $A_{1}$ whose complement $B_{1}$ belongs to $\mathcal{u}$. 
Proof. Let $B$ be the closure of $B_{1}$ in $u$ then if $A, A_{1}$ are complements, respectively, of $B$ and $B_{1}, A \subset A_{1}$, and so $\left(T_{A} P T_{A}\right)^{n} 1$ $\leqq\left(T_{A_{1}} P T_{A_{1}}\right)^{n 1}$ and if the right-hand side converges to zero so does the left-hand side. Conversely let $B_{1} \in \mathcal{U}$; find an open set $B_{0} \in \mathcal{U}$ with $B_{0} \subset \operatorname{cl}\left(B_{0}\right) \subset B_{1}$. Put $A$ as the complement of $\operatorname{cl}\left(B_{0}\right)$ and $A_{1}$ the complement of $B_{1}$ then $A_{1} \subset A$ and $\left(T_{A_{1}} P T_{A_{1}}\right)^{n} 1 \leqq\left(T_{A} P T_{A}\right)^{n} 1$.

As in [3, Lemma 2] it is easy to show that if (4) holds for every set $A$, whose complement is in $\mathcal{U}$ then $\sum_{n=1}^{\infty} P^{n} 1_{B}(x)>0$ for every $x \in X$ and $B \in \mathcal{~}$. Thus if $\lambda$ is any $\sigma$ finite countably additive invariant measure then $\lambda(B)>0$ for every $B \in \mathcal{~}$.

Theorem 3. Let $P$ satisfy (1), (2) and (3). The operator $P$ satisfies (4), for every complement of a set in $u$, if and only if

(5) If $g$ is upper semicontinuous, $0 \leqq g \leqq 1$, and $P g \geqq g$, then $g$ is a constant.

Proof. If condition (4) fails for some $A$ whose complement $B \in \mathcal{U}$, let $\alpha$ be a continuous function with $0 \leqq \alpha \leqq 1, \alpha \geqq 1_{A}$ and $\alpha=0$ on some set $C, C \in \mathcal{u}$. Then $(\alpha P \alpha)^{n} 1 \downarrow g$ where $g=0$ on $C, g$ is not identically zero. Now $g=\alpha P \alpha g \leqq P(\alpha g) \leqq P g$ and $g$ is upper semicontinuous which contradicts (5). Conversely, if $g$ is upper semicontinuous and $P g \geqq g$ find some $0<a<1$ so that $\{x: g(x)<a\}$ is an open set which is neither $\phi$ nor $X$. This is possible since $g$ is not a constant. Put $g_{1}=\max (g-a, 0)$ then $g_{1}$ is again upper semicontinuous, $P g_{1} \geqq g_{1}$ and $\left\{x: g_{1}(x)=0\right\}$ contains an open set, $V$, in $\mathcal{U}$. Let $A$ be the complement of $V$ then $\left(T_{A} P T_{A}\right) g_{1}=T_{A} P g_{1} \geqq T_{A} g_{1}=g_{1}$ so $\left(T_{A} P T_{A}\right)^{n} 1 \geqq\left(T_{A} P T_{A}\right)^{n} g_{1} \geqq g_{1}$ contradicting (4).

\section{REFERENCES}

1. N. Dunford and J. Schwartz, Linear operators, Interscience, New York, 1958.

2. S. R. Foguel, Invariant measures for Markov processes. II, Proc. Amer. Math. Soc. 17 (1966), 387-389.

3. - Existence of a $\sigma$ finite invariant measure for a Markov process on a locally compact space, Israel J. Math. 6 (1968), 1-4.

4. K. Yosida and E. Hewitt, Finitely additive measures, Trans. Amer. Math. Soc. 72 (1952), 46-66.

The University of Minnesota and The Hebrew University of Jerusalem 